\title{
Pozzolanic activity of oil-refining catalyst: evaluation by electron and atomic force microscopy
}

Jeremias, J., , Nogueira, I. , Nolasco, P. , Carvalho, P.A. , Ferreira, C. ${ }^{* *}$, Costa, C. ${ }^{* *}$

* ICEMS, Instituto Superior Técnico, University of Lisbon, Av. Rovisco Pais, 1049-001 Lisbon, Portugal

** Civil Engineering Department, Instituto Superior de Engenharia de Lisboa, R. Conselheiro Emídio Navarro, 1959-007 Lisbon, Portugal

Email : pac@ist.utl.pt

The reuse of waste fluid catalytic cracking (FCC) catalyst as partial surrogate for cement can reduce the environmental impact of both the oil-refinery and cement production industries $[1,2]$. FCC catalysts can be considered as pozzolanic materials since in the presence of water they tend to chemically react with calcium hydroxide to produce compounds possessing cementitious properties [3,4]. In addition, partial replacement of cement with FCC catalysts can enhance the performance of pastes and mortars, namely by improving their compressive strength $[5,6]$.

In the present work the reaction of waste $\mathrm{FCC}$ catalyst with $\mathrm{Ca}(\mathrm{OH})_{2}$ has been investigated after a curing time of 28 days, by scanning electron microscopy (SEM) with electron backscattered signal (BSE) combined with X-ray energy dispersive spectroscopy (EDS) carried out with a JEOL JSM 7001F instrument operated at $15 \mathrm{kV}$ coupled to an INCA pentaFetx3 Oxford spectrometer. The polished cross-sections of FCC particles embedded in resin have also been evaluated by atomic force microscopy (AFM) in contact mode (CM) using a NanoSurf EasyScan 2 instrument.

The SEM/EDS results revealed that an inward migration of $\mathrm{Ca}$ occurred during the reaction. A weaker outward migration of $\mathrm{Si}$ and $\mathrm{Al}$ was also apparent (Fig. 1). The migration of $\mathrm{Ca}$ was not homogeneous and tended to follow high-diffusivity paths within the porous waste FCC catalyst particles. The present study suggests that the porosity of waste FCC catalysts is key for the migration/reaction of Ca from the surrounding matrix, playing an important role in the pozzolanic activity of the system. The topography images and surface roughness parameters obtained by atomic force microscopy can be used to infer the local porosity in waste FCC catalyst particles (Fig. 2).

The authors acknowledge financial support of the Portuguese Foundation for Science and Technology through PTDC/ECM/113115/2009 and PEst-OE/CTM-UI0084/2011 grants.

Communication submitted to the Microscopy at the Frontiers of Science 2013 congress in Tarragona, Spain.

[1] Su, N. et al., Cement and Concrete Research, 30, 1773-1783, 2000.

[2] Hsu, K.C., Cement and Concrete Research, 31, 1815-1820, 2001.

[3] Pacewska, B. et al., Cement and Concrete Research, 32, 145-152, 2002.

[4] Paya, J. et al., Cement and Concrete Research, 33, 603-609, 2003.

[5] Wu, I. et al., Cement and Concrete Research, 33, 245-253, 2003.

[6] Costa, C. et al., Proc. of ICDC 2012 International Congress on Durability of Concrete CD-ROM, Trondheim, Norway, June 2012. 


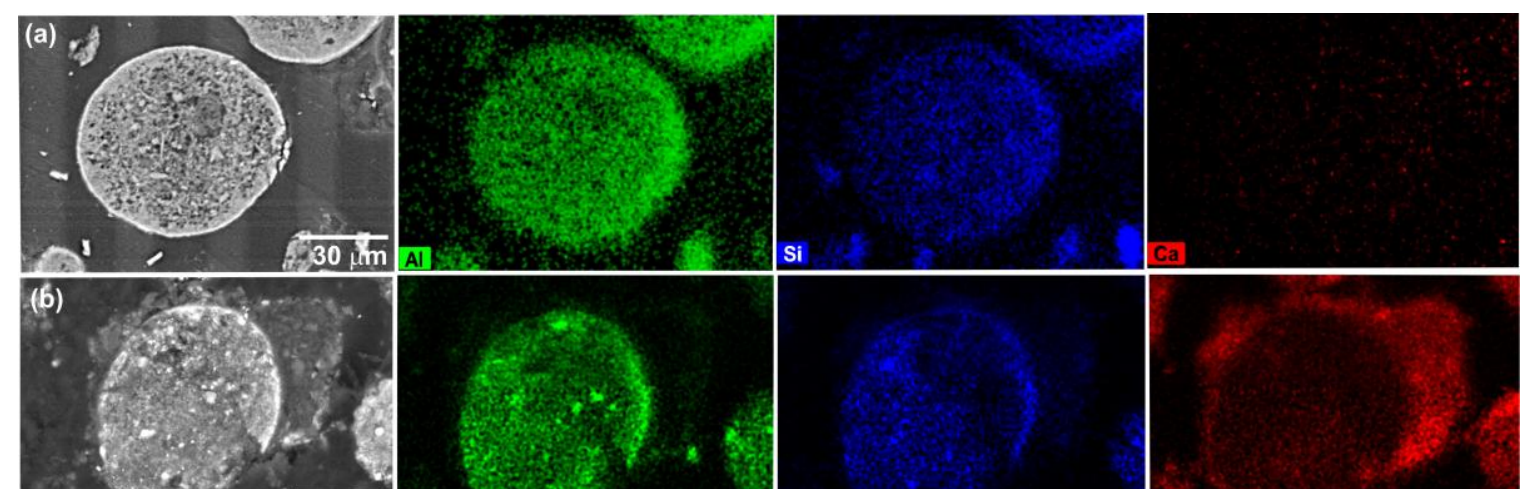

Figure 1. BSE-SEM images and corresponding X-ray maps of (a) waste FCC catalyst and (b) after 28 days in the presence of $\mathrm{Ca}(\mathrm{OH})_{2}$. This phase is visible around the prior FCC particles.
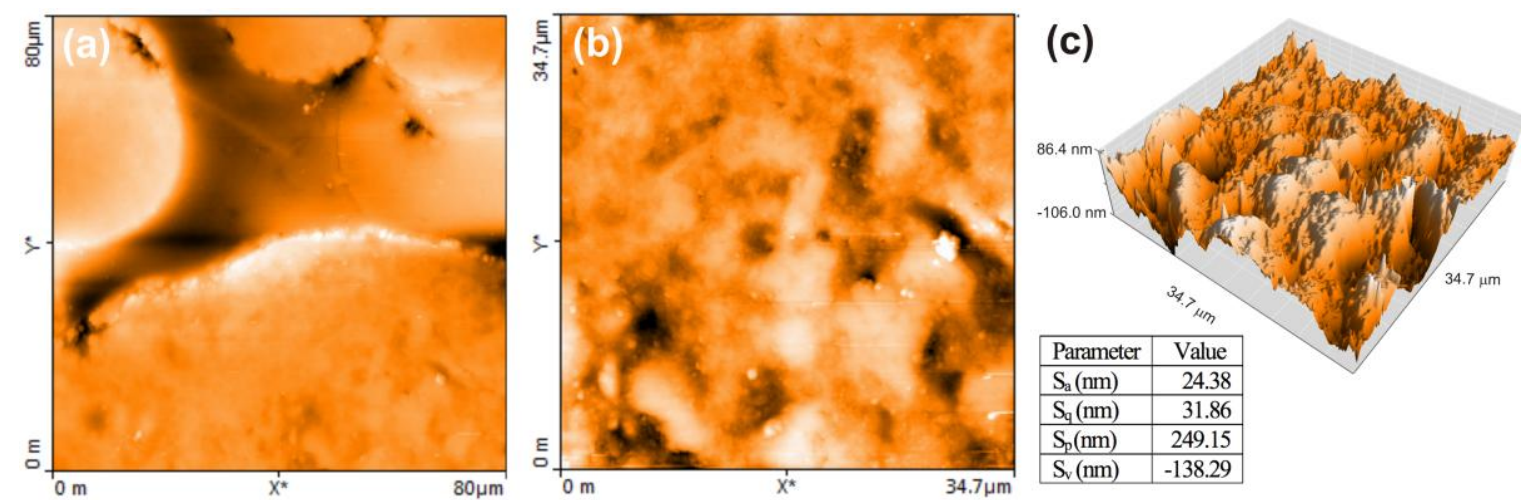

Figure 2. AFM-CM images of (a) polished sections of FCC catalyst particles embedded in epoxy resin. (b) Magnified detail of a waste FCC catalyst particle and (c) corresponding 3-D rendering with a list of the surface roughness parameters. 